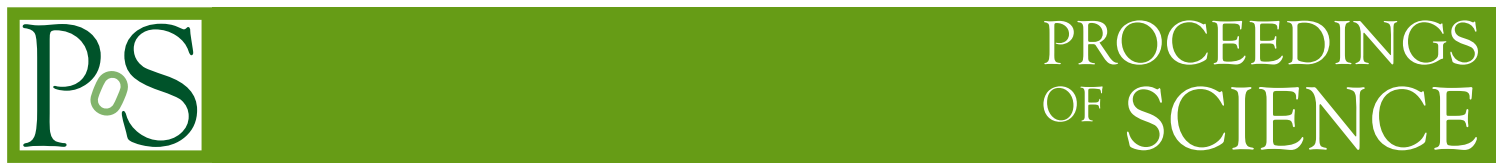

\title{
Electron in a transverse harmonic potential
}

\section{H. Honkanen ${ }^{* \dagger}$}

Iowa State University, USA

E-mail: heli@iastate.edu

\begin{abstract}
Non-perturbative solutions to the quantum-field theory is a topic of current and broad interest, especially for the heavy ion and laser physics communities, since they investigate particle production in the presence of strong external fields. We have solved a non-perturbative QED + external field problem of an electron in a strong transverse confining potential using Hamiltonian light-front quantum field theory in a basis function representation. The invariant mass spectra and the anomalous magnetic moment of the lowest state for this two-scale system are also evaluated. With this method the perturbative QED results are also reproduced with a good accuracy. We also discuss the extension of the method to other problems as well.
\end{abstract}

Light Cone 2010 - LC2010

June 14-18, 2010

Valencia, Spain

* Speaker.

${ }^{\dagger}$ This work was done in collaboration with P. Maris, J. P. Vary and S. J. Brodsky, and was supported in part by a DOE Grant DE-FG02-87ER40371. Computational resources were provided by DOE through the National Energy Research Supercomputer Center (NERSC). 


\section{Motivation}

The Hamiltonian light-front formalism is ideal for solving problems non-perturbatively, since time is set along the light-front and evaluated experimental observbles, such as masses, form factors, and structure function, are Lorentz frame independent. Light-front Hamiltonian quantum field theory also has similarities with non-relativistic quantum many-body theory. This connection was exploited in [1], where a "Basis Light Front Quantized (BLFQ)" approach was outlined by adopting a light-front single-particle basis space consisting of the 2-D harmonic oscillator for the transverse modes and a discretized momentum space basis for the longitudinal modes. Adoption of this basis is also consistent with recent developments in AdS/CFT correspondence with QCD [2].

In [3] this approach was applied to address the problem of an electron in a transverse harmonic cavity with the QED Hamiltonian evaluated on the light-front in a Fock space consisting of electron states and electron plus photon states. The external field was included non-perturbatively and the eigenvalues, eigenvectors and anomalous magnetic moment were solved. In a non-renormalized case, the obtained electron anomalous magnetic moment was within $1.5 \%$ of the theoretically expected result (Schwinger moment), when extrapolated to the zero external field limit. Applying a sector-dependent renormalization scheme [4] to this Hamiltonian, the zero external field results were consistent with related works Refs.[5, 6] and Refs.[7, 8], where the one-photon truncated light-front Hamiltonian was regulated with a Pauli-Villars regularization scheme.

The nonperturbative analysis presented in [3] could be applicable to measurements of the (gyromagnetic) ratio of the spin precession to Larmor frequencies of a trapped electron in strong external electromagnetic fields, and can be straightforwardly extended by incorporating higher Fock-space sectors. It also serves as a first step towards the studies of non-perturbative QED relevant for the anomalous enhancement of lepton production at RHIC [9] and for proposals for producing super-critical fields with next-generation laser facilities [10,11]. Another direction for QED applications follows the lines of $[12,13]$, where Fourier transform of the Deeply Virtual Compton Scattering amplitude with respect to the skewness variable $\zeta$ at fixed invariant momentum transfer was observed to be analogous to the diffractive scattering of a wave in optics. In analogy with this "hadron optics", the light-front electron wave functions computed in [3] can be used to evaluate the form factors of the electron and thereby introduce "electron optics".

Most importantly, the research in [1,3] also serves as a foundation for solving other quantum field theories at strong coupling, such as the light-front QCD Hamiltonian in the nonperturbative domain. In order to extend the research to QCD, methods for treating the color degree of freedom in a computationally efficient manner were already introduced and evaluated in [1] . The next step is then to incorporate the color degree of freedom into the quantum field theory code, that has already passed an important accuracy test in [3].

We will next review the method used in [1,3], adding some details not previously discussed.

\section{BLFQ Hamiltonian Framework}

In BLFQ approach the Hamiltonian is expressed in terms of basis functions, and the size of the resulting Hamiltonian matrix is regulated by imposing both physical symmetries and different cut-

off conditions for the basis states. Increasing the size of the basis will inevitably lead to substantial 
computational requirements both in the computation of the matrix elements themselves and in the diagonalization of the matrix. In order to be able to extrapolate the results to the continuum limit, where the cut-offs are removed, a rapid convergence of the results is then highly desirable.

We define our light-front coordinates as $x^{ \pm}=x^{0} \pm x^{3}, x^{\perp}=\left(x^{1}, x^{2}\right)$, where the variable $x^{+}$ is light-front time and $x^{-}$is the longitudinal coordinate. We adopt $x^{+}=0$, the "null plane", for our quantization surface. In our choice of framework [1,3] we quantize QED on the light-front using the light-front gauge, and add the harmonic oscillator potential in the transverse direction to confine the system in those directions. To simplify the numerical work, we choose the transverse basis function scale and the trap scale to coincide. As a consequence, we cannot obtain zero external field QED results directly, but via extrapolation, as shown later.

Our basis states consist of 2-D harmonic oscillator (HO) states, which are combined with discretized longitudinal modes, plane waves satisfying selected boundary conditions. The HO states are characterized by a principal quantum number $n$, orbital quantum number $m$ and $\mathrm{HO}$ energy $\Omega$. We express the 2-D oscillator in momentum space as a function of the dimensionless variable $\rho=\left|p^{\perp}\right| / \sqrt{M_{0} \Omega}$, where $M_{0}$ has units of mass. The orthonormalized HO wavefunctions in polar coordinates $(\rho, \varphi)$ are then given in terms of the Generalized Laguerre Polynomials, $L_{n}^{|m|}\left(\rho^{2}\right)$, by

$$
\Phi_{n m}\left(p^{\perp}\right)=\Phi_{n m}(\rho, \varphi)=\langle\rho \varphi \mid n m\rangle=\sqrt{\frac{2 \pi}{M_{0} \Omega}} \sqrt{\frac{2 n !}{(|m|+n) !}} e^{i m \varphi} \rho^{|m|} e^{-\rho^{2} / 2} L_{n}^{|m|}\left(\rho^{2}\right),
$$

with eigenvalues $E_{n, m}=(2 n+|m|+1) \Omega$. These wavefunctions are orthogonal and form a complete set of states, thus

$$
\sum_{n m} \Phi_{n m}^{*}\left(p^{\perp}\right) \Phi_{n m}\left(q^{\perp}\right)=(2 \pi)^{2} \delta^{(2)}\left(p^{\perp}-q^{\perp}\right) .
$$

The longitudinal modes, $\psi_{k}$, in our basis are defined for $-L \leq x^{-} \leq L$ with periodic boundary conditions (PBC) for the photon and antiperiodic boundary conditions (APBC) for the electron:

$$
\psi_{k}\left(x^{-}\right)=\frac{1}{\sqrt{2 L}} \mathrm{e}^{i \frac{\pi}{L} k x^{-}},
$$

where $k=1,2,3, \ldots$ for PBC (we neglect the zero mode) and $k=\frac{1}{2}, \frac{3}{2}, \frac{5}{2}, \ldots$ for APBC. The full 3-D single-particle basis state is defined by the product form

$$
\Psi_{k, n, m}\left(x^{-}, \rho, \varphi\right)=\psi_{k}\left(x^{-}\right) \Phi_{n, m}(\rho, \varphi) .
$$

Following Ref.[14] we introduce the total invariant mass-squared $M^{2}$ for the low-lying physical states in terms of a Hamiltonian $H$ times a dimensionless integer for the total light-front momentum $K$

$$
M^{2}+P_{\perp} P_{\perp} \rightarrow M^{2}+\text { const }=P^{+} P^{-}=K H
$$

where we absorb the constant into $M^{2}$. The non-interacting Hamiltonian $H_{0}$ for this system (where now the transverse functions for both the fermion and the boson are taken as eigenmodes of the trap) is then defined by the sum of the occupied modes $i$ in each many-parton state as

$$
H_{0}=2 M_{0} P_{c}^{-}=\frac{2 M_{0} \Omega}{K} \sum_{i} \frac{2 n_{i}+\left|m_{i}\right|+1+\bar{m}_{i}^{2} /\left(2 M_{0} \Omega\right)}{x_{i}},
$$


where $\bar{m}_{i}$ is the mass of the parton $i$. The photon mass is always set to zero in the following and the electron mass $\bar{m}_{e}$ is set at the physical mass $0.511 \mathrm{MeV}$ in our non-renormalized calculations. We also set $M_{0}=\bar{m}_{e}$.

The basis is limited to fermion and fermion-boson states, so the light-front QED Hamiltonian interaction terms we need are the fermion to fermion-boson vertex, given as

$$
V_{e \rightarrow e \gamma}=\left.g \int d x_{+} d^{2} x_{\perp} \bar{\Psi}(x) \gamma^{\mu} \Psi(x) A_{\mu}(x)\right|_{x^{+}=0},
$$

and the instantaneous fermion-boson interaction,

$$
V_{e \gamma \rightarrow e \gamma}=\left.\frac{g^{2}}{2} \int d x_{+} d^{2} x_{\perp} \bar{\Psi} \gamma^{\mu} A_{\mu} \frac{\gamma^{+}}{i \partial^{+}}\left(\gamma^{v} A_{\nu} \Psi\right)\right|_{x^{+}=0},
$$

where the coupling constant $g^{2}=4 \pi \alpha$, and $\alpha$ is the fine structure constant taken to be $\alpha=\frac{1}{137.036}$. When expressing the free fermion and boson fields in terms of our basis functions, the complete set of quantum numbers needed to specify a state are $\bar{\alpha}=(k, n, m, \lambda)$, where $\lambda$ is the helicity. The fermion and boson annihilation operators are then written as

$$
\begin{aligned}
& b\left(p^{\perp}, \alpha\right)=\sum_{n m} b(\bar{\alpha}) \Phi_{n m}\left(p^{\perp}\right), \\
& a\left(p^{\perp}, \gamma\right)=\sum_{n m} a(\bar{\gamma}) \Phi_{n m}\left(p^{\perp}\right),
\end{aligned}
$$

where

$$
\begin{aligned}
& {\left[a(\bar{\gamma}), a^{\dagger}(\bar{\gamma})\right]=\delta_{\bar{\gamma}}^{\bar{\gamma}^{\prime}},} \\
& \left\{b(\bar{\alpha}), b^{\dagger}\left(\bar{\alpha}^{\prime}\right)\right\}=\delta_{\bar{\alpha}}^{\bar{\alpha}^{\prime}},
\end{aligned}
$$

and the truncated set of quantum numbers $\alpha=(k, \lambda)$. After this replacement and proper normalization, the non-spinflip vertex terms of Eq.(2.7) are $\propto M_{0} \Omega$, similar to the non-interacting Hamiltonian of Eq.(2.6), whereas spinflip terms are $\propto \sqrt{M_{0} \Omega} m_{e}$. Selecting the initial state fermion helicity in the single fermion sector always as "up", the process $e \rightarrow e \gamma$ is nonzero for 3 out of 8 helicity combinations, and the process $e \gamma \rightarrow e \gamma$ is nonzero only when all 4 spin projections aligned ( 2 out of 16 combinations). The resulting Hamiltonian matrix is thus sparse.

As a symmetry constraint for the basis we fix the total angular momentum projection $J_{z}=$ $M+S=\frac{1}{2}$, where $M=\sum_{i} m_{i}$ is the total azimuthal quantum number, and $S=\sum_{i} s_{i}$ the total spin projection along the $x^{-}$direction. For cutoffs, we select the total light-front momentum, $K$, and the maximum total quanta allowed in the transverse mode of each one or two-parton state, $N_{\max }$, such that

$$
\begin{aligned}
& \sum_{i} x_{i}=1=\frac{1}{K} \sum_{i} k_{i}, \\
& \sum_{i} 2 n_{i}+\left|m_{i}\right|+1 \leq N_{\max },
\end{aligned}
$$

where, for example, $k_{i}$ defines the longitudinal modes of Eq.(2.3) for the $i^{\text {th }}$ parton. Eq.(2.13) signifies total light-front momentum conservation written in terms of boost-invariant momentum 
fractions, $x_{i}$. Since each parton carries at least one unit of longitudinal momentum, the basis is limited to $K$ partons. Furthermore, since each parton carries at least one oscillator quanta for transverse motion, the basis is also limited to $N_{\max }$ partons. Thus the combined limit on the number of partons would be $\min \left(K, N_{\max }\right)$, if the Fock space was not truncated.

\section{BLFQ Hamiltonian results}

Here we present some numerical results from [3] for cases where the cutoffs for the basis space dimensions are selected such that $K$ increases simultaneously with the $N_{\max }$. The resulting dimension of the Hamiltonian matrix increases rapidly. For $N_{\max }=K=2,10,20$, the dimensions of the corresponding symmetric $d \times d$ matrices are $d=2,1670,26990$, respectively. Since we employ a mix of boundary conditions and all states have half-integer total $K$, we quote $K$-values rounded downwards for convenience, except when the precise value is required.
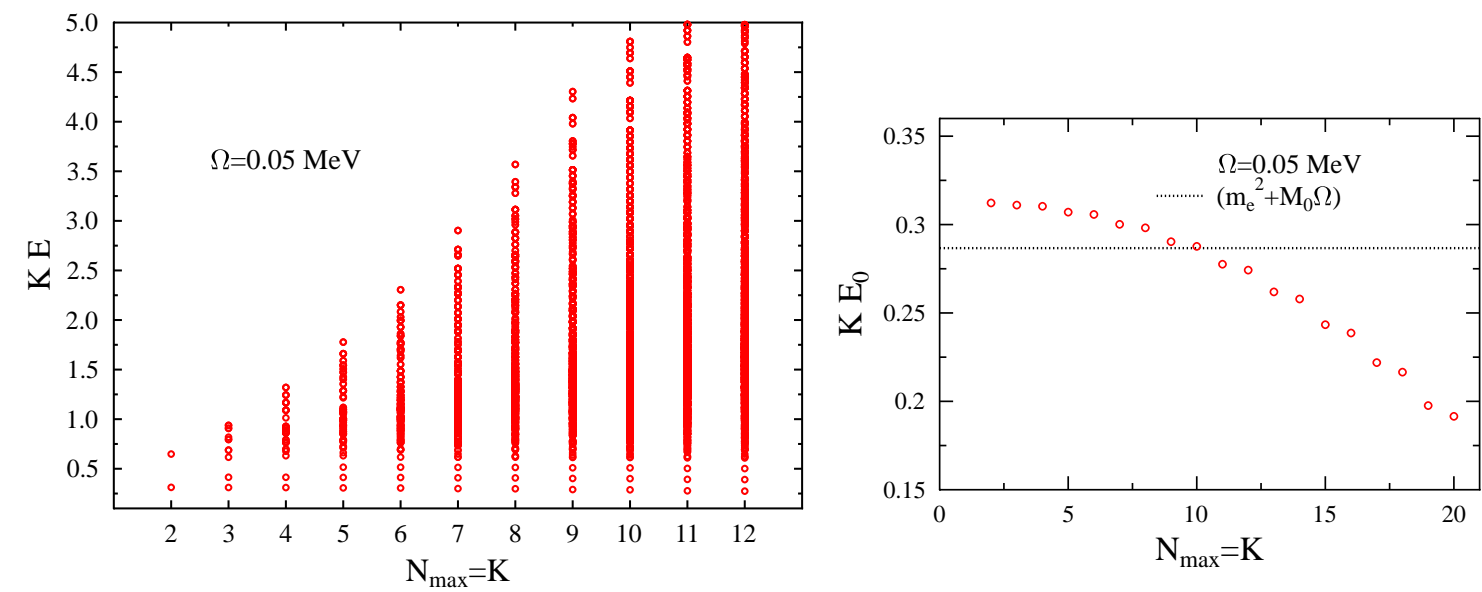

Figure 1: Eigenvalues (multiplied by $K$ ) for a non-renormalized light-front QED Hamiltonian which includes the fermion-boson vertex and the instantaneous fermion-boson interaction without counterterms (figure adapted from [3]). Closeup of the lowest-lying eigenvalues on the right panel. The basis is limited to fermion and fermion-boson states satisfying the symmetries. The cutoffs for the basis space dimensions are selected such that $K$ increases simultaneously with the $N_{\max }$.

In the left panel of Fig.1 we show the eigenvalues (multiplied by $K$ ) for a non-renormalized light-front QED Hamiltonian given in Eqs.(2.6,2.7,2.8), with fixed $\Omega=0.05 \mathrm{MeV}$. In the right panel we show a closeup of the lowest-lying eigenvalues only. These eigenvalues correspond to a solution dominated by the electron with $n=m=0$, and are expected to be $K E_{0}=m_{e}^{2}+M_{0} \Omega$, where the latter term accounts for the lowest state of transverse motion of the electron allowed in the chosen basis. In Fig.1 the lowest eigenvalues for a fixed $\Omega$ fall below that value, as the size of the matrix increases. The contribution of the spinflip terms to the lowest eigenvalues is very small, and, as a result, the lowest eigenvalues for a fixed $N_{\max }=K$ depend linearly on $\Omega$. Since our system is in an external field, the lowest physical mass eigenstate (not known experimentally) can deviate from the free-space mass. Therefore, before renormalization, we choose only to consider cases where the mass eigenvalue falls within $25 \%$ of the free electron mass. 
The ordering of excited states in Fig.1, due to significant interaction mixing, does not always follow the highly degenerate unperturbed spectrum of Eq.(2.6). States dominated by spin-flipped electron-photon components are evident in the solutions. Nevertheless, the lowest-lying eigenvalues appear with nearly harmonic separations in Fig.1 as would be expected at the coupling of QED. The multiplicity of the higher eigenstates increases rapidly with increasing $N_{\max }=K$ and the states exhibit stronger mixing with other states than the lowest-lying states. In principle the fermion-boson basis states interact directly with each other in leading order through the instantaneous fermion-boson interaction, but numerically the effect of this interaction is very weak, and thus does not contribute significantly to the mixing. Even though we work within a Fock space approach, our numerical results should approximately equal the lowest order perturbative QED results for sufficiently weak external field.

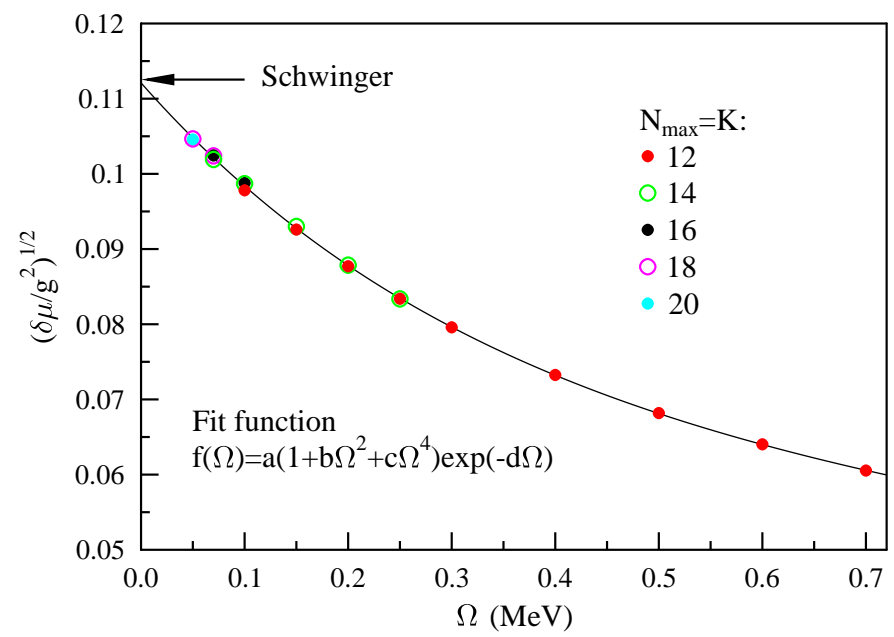

Figure 2: (Color online) Square root of the (scaled) electron anomalous magnetic moment as a function of the transverse external field for a sequence of increasing basis spaces indicated in the legend. These are nonrenormalized results where the mass eigenvalue falls within $25 \%$ of the free electron mass. Extrapolation to zero external field yields 0.1121, compared with the theoretical 1-loop QED prediction ("Schwinger") of 0.1125 . Figure is adapted from [3].

In Fig. 2 we show the results for the square root of the electron anomalous magnetic moment (scaled), $\sqrt{\delta \mu / g^{2}}$, as a function of $\Omega$ obtained from the lowest mass eigenstate. That is, we plot the magnitude of the probability amplitude that the electron has its spin flipped relative to the single electron Fock-space component in the range where the results are converged. For even $N_{\max }=K$ the results converge rapidly for $N_{\max }=K \geq 14$. The results for odd cutoffs (not shown) track even cutoff results as $N_{\max }=K$ increases. Below $\Omega \lesssim 0.05 \mathrm{MeV}$ (results not shown), in the weak external field region, all the interactions are quenched at fixed $N_{\max }=K$, and not converged, due in part to our requirement that the $\mathrm{HO}$ basis states track the external field. In order to compare our results with the square root of the ratio of the Schwinger result $\frac{\alpha}{2 \pi}$ for the coupling constant $g^{2}=4 \pi \alpha$, we perform an extrapolation of the above results for $N_{\max }=K=12, \ldots, 20$ to the zero external field limit $\Omega=0 \mathrm{Mev}$. An excellent agreement with the results is obtained by a fit function $f(\Omega)=$ $a\left(1+b \Omega^{2}+c \Omega^{4}\right) \exp (-d \Omega)$, with $a=0.1121$. This is $<1 \%$ deviation from the Schwinger result of 0.1125 , which is reasonable in light of our numerical accuracy and extrapolation uncertainties. 


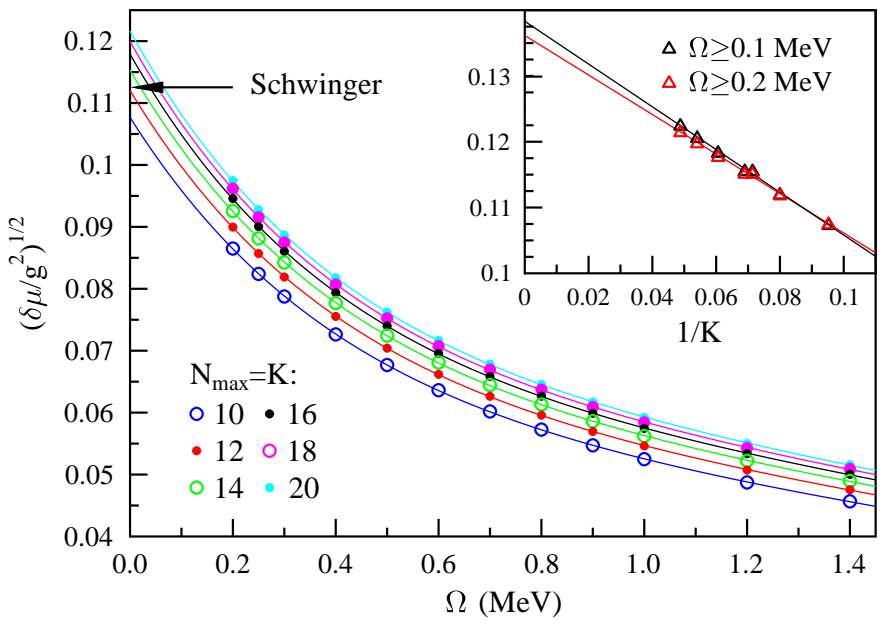

Figure 3: (Color online) Individual fits to the renormalized results for square root of the (scaled) electron anomalous magnetic moment for $N_{\max }=K=10, \ldots, 20$. The inset shows the continuum limit extrapolation of the zero external field results from the main panel as a function of $1 / K$. Figure is adapted from [3].

In Fig. 3 we renormalize our results for $\sqrt{\delta \mu / g^{2}}$ by applying a sector-dependent normalization scheme from Ref.[4]. In our present limited Fock space, we need only the mass counterterm $\delta m_{e}$. This $\delta m_{e}$ is added to the mass term in the diagonal one-electron part of the Hamiltonian Eq.(2.6). In the absence of a known experimental mass for renormalization due to the external field, we adjust $\delta m_{e}$ such that the lowest eigenstate remains at $K E_{0}=m_{e}^{2}+M_{0} \Omega$. To eliminate possible effects from the quenched interactions at small external fields, we only include results with the external field $\Omega \geq 0.2 \mathrm{MeV}$. Again, individual fits of the form given Fig.2 in are an excellent representation of our results. The range of the extrapolated values is $0.1077 \leq a \leq 0.1216$.

The convergence with an increasing cutoff is now less rapid than in the non-renormalized case shown Fig.2. In order to approach the continuum limit $N_{\max }=K \rightarrow \infty$, we perform further extrapolation to the zero- $\Omega$ results of Fig.3. The inset of Fig.3 shows linear extrapolation of the results of the main figure in $1 / K$ to the continuum limit $N_{\max }=K \rightarrow \infty$. To verify the stability of the results, an extrapolation based on the $\Omega \geq 0.1 \mathrm{MeV}$ fits (not shown) is also given. The extrapolated continuum values are $0.1362(0.1383)$ for $\Omega \geq 0.2(0.1)$, respectively, and thus about $20 \%$ above the Schwinger result 0.1125. As mentioned in Sec.1, an enhancement of this magnitude was also observed in related works, Ref.[6] and Refs.[7, 8], where one-photon truncated light-front Hamiltonian was regulated with Pauli-Villars (PV) regularization scheme. With PV regularization as well as in our renormalized results, interpreted from a perturbation theory perspective, the intermediate state propogators are developed from a dynamical (non-perturbative) electron mass rather than using the unperturbed mass needed for direct comparison with perturbation theory. Thus one may appreciate why the renormalized results are distinct from the lowest order perturbative results.

The extension of this method both to a larger Fock-space and to QCD will proceed as outlined in [1]. In the case of non-interacting QED, the state density as a function of the state energy $E$, given by Eq.(2.6), was found to increase exponentially as $N_{\max }=K$ increases. Similarly, implementation of the color dramatically increases the state density over the case of QED, as shown in Fig.4, but it 
was also found, that use of a global color-singlet constraint is effective in minimizing the explosion in basis space states. We anticipate to be able to handle both of these challenges with the parallel codes developed, tested and applied in [1,3], that compute the Hamiltonian matrix and solve for its eigenvalues and eigenvectors

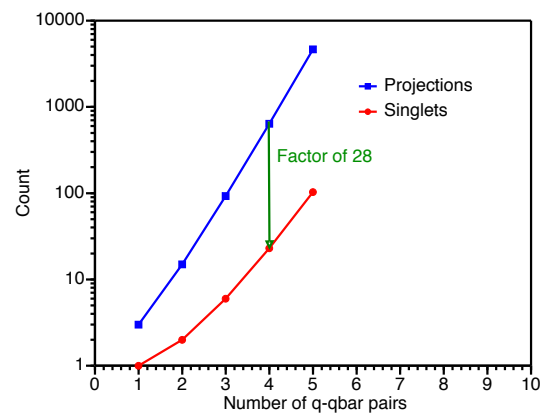

Figure 4: Number of color space states that apply to space-spin configuration of selected multi-parton states for two methods of enumerating the color basis states. The upper curve shows counts of all color configurations with zero color projection. The lower curve counts global color singlets. Figure adapted Ref.[1].

\section{References}

[1] J. P. Vary et al., Phys. Rev. C 81,(2010) 035205.

[2] G. F. de Teramond and S. J. Brodsky, Phys. Rev. Lett. 102, 081601 (2009).

[3] H. Honkanen, P. Maris, J. P. Vary and S. J. Brodsky, arXiv:1008.0068 [hep-ph].

[4] V. A. Karmanov, J. F. Mathiot and A. V. Smirnov, Phys. Rev. D 77 (2008) 085028.

[5] S. J. Brodsky, J. R. Hiller and G. McCartor, Phys. Rev. D 58, 025005 (1998).

[6] S. J. Brodsky, V. A. Franke, J. R. Hiller, G. McCartor, S. A. Paston and E. V. Prokhvatilov, Nucl. Phys. B 703 (2004) 333.

[7] S. S. Chabysheva and J. R. Hiller, arXiv:0911.3686 [hep-ph].

[8] S. S. Chabysheva and J. R. Hiller, Phys. Rev. D 81 (2010) 074030.

[9] A. Adare et al. [PHENIX Collaboration], Phys. Rev. C 81 (2010) 034911.

[10] M. Ruf, G. R. Mocken, C. Muller, K. Z. Hatsagortsyan and C. H. Keitel, Phys. Rev. Lett. 102 (2009) 080402 .

[11] C. K. Dumlu and G. V. Dunne, Phys. Rev. Lett. 104 (2010) 250402, and references therein.

[12] S. J. Brodsky, D. Chakrabarti, A. Harindranath, A. Mukherjee and J. P. Vary, Phys. Lett. B 641 (2006) 440 [arXiv:hep-ph/0604262].

[13] S. J. Brodsky, D. Chakrabarti, A. Harindranath, A. Mukherjee and J. P. Vary, Phys. Rev. D 75 (2007) 014003 [arXiv:hep-ph/0611159].

[14] S. J. Brodsky, H. C. Pauli and S. S. Pinsky, Phys. Rept. 301 (1998) 299. 